\title{
Mitochondrial abnormalities related to the dysfunction of circulating endothelial colony-forming cells in moyamoya disease
}

\author{
*Jung Won Choi, MD, ${ }^{1}$ Sung Min Son, PhD, ${ }^{2}$ Inhee Mook-Jung, PhD, ${ }^{2}$ Youn Joo Moon, MS, ${ }^{3,4}$ \\ Ji Yeoun Lee, MD, PhD, ,-5 Kyu-Chang Wang, MD, PhD, ${ }^{3,4}$ Hyun-Seung Kang, MD, PhD, ${ }^{4}$ \\ Ji Hoon Phi, MD, PhD, ${ }^{3,4}$ Seung Ah Choi, PhD, ${ }^{3,4}$ Sangjoon Chong, MD, ${ }^{3,4}$ Jayoung Byun, MS, ${ }^{2}$ and \\ Seung-Ki Kim, MD, PhD 3,4

\begin{abstract}
${ }^{1}$ Department of Neurosurgery, Samsung Medical Center, Sungkyunkwan University School of Medicine; ${ }^{2}$ Department of Biochemistry and Biomedical Sciences; 'Division of Pediatric Neurosurgery, Seoul National University Children's Hospital; ${ }^{4}$ Department of Neurosurgery, Seoul National University Hospital; and ${ }^{5}$ Department of Anatomy and Cell Biology, Seoul National University College of Medicine, Seoul, Republic of Korea
\end{abstract}

\begin{abstract}
OBJECTIVE Moyamoya disease (MMD) is a unique cerebrovascular disorder characterized by the progressive occlusion of the bilateral internal carotid arteries. Endothelial colony-forming cells (ECFCs), previously termed "endothelial progenitor cells," play an important role in the pathogenesis of MMD. In this study, the authors performed morphological and functional studies of the mitochondria of ECFCs from patients with MMD to present new insights into the pathogenesis of the disease.
\end{abstract}

METHODS The morphology of ECFCs from 5 MMD patients and 5 healthy controls was examined under both a transmission electron microscope and a confocal laser scanning microscope. The oxygen consumption rates (OCRs), mitochondrial membrane potentials (MMPs), intracellular $\mathrm{Ca}^{2+}$ concentrations, mitochondrial enzyme activities, and reactive oxygen species (ROS) levels were measured. Functional activity of the ECFCs was evaluated using a capillary tube formation assay.

RESULTS The ECFCs from the MMD patients displayed a disrupted mitochondrial morphology, including a shorter and more circular shape. The ECFC mitochondria from the MMD patients exhibited functional abnormalities, which were assessed as a decreased OCR and an increased intracellular $\mathrm{Ca}^{2+}$ concentration. Moreover, the ECFCs from MMD patients showed increased ROS levels. Interestingly, treatment with an ROS scavenger not only reversed the mitochondrial abnormalities but also restored the angiogenic activity of the ECFCs from the MMD patients.

CONCLUSIONS The mitochondria of ECFCs from MMD patients, as compared with those from healthy patients, exhibited morphological and functional abnormalities. This finding suggests that the mitochondrial abnormalities may have a role in the pathogenesis of MMD.

https://thejns.org/doi/abs/10.3171/2017.5.JNS17147

KEY WORDS cerebrovascular disease; mitochondria; moyamoya; vasculogenesis; cell culture; vascular disorders

$\mathrm{M}$ OYAMOYa disease (MMD) is a unique cerebrovascular disorder of unknown cause, characterized by the progressive bilateral occlusion of the internal carotid artery and its major branches with the arterial collateral vessels at the base of the brain. It is well established that this disease has a higher prevalence in
East Asia and that $10 \%-15 \%$ of patients with MMD have a family history of the disease. ${ }^{12}$ Moreover, it is well known that MMD occurs more frequently among women. ${ }^{12}$ This information suggests that genetic factors participate in the etiology of MMD.

Therefore, diverse genetic analyses of MMD have at-

ABBREVIATIONS ATP = adenosine triphosphate; $\left[\mathrm{Ca}^{2+}\right]_{\mathrm{i}}=$ intracellular $\mathrm{Ca}^{2+}$ concentration; $\mathrm{CCCP}=$ carbonyl cyanide $\mathrm{m}-$ chlorophenyl hydrazine; $\mathrm{DCFH}-\mathrm{DA}=2^{\prime}, 7^{\prime}$-dichlorodihydrofluorescein diacetate; ECFC = endothelial colony-forming cell; EPC = endothelial progenitor cell; Fluo-4 = fluo-4 acetoxymethyl ester; MMD = moyamoya disease; MMP = mitochondrial membrane potential; MTT = 3-(4,5-dimethylthiazol-2-yl)-2,5-diphenyltetrazolium bromide; NAC = N-acetyl-L-cysteine; OCR = oxygen consumption rate; PBS = phosphate-buffered saline; ROS = reactive oxygen species; TMRM = tetramethylrhodamine methyl ester.

SUBMITTED January 16, 2017. ACCEPTED May 8, 2017.

INCLUDE WHEN CITING Published online December 8, 2017; DOI: 10.3171/2017.5.JNS17147.

* J. W. Choi and S. M. Son contributed equally to this work. I. Mook-Jung and S. K. Kim share senior authorship of this work. 
tempted to uncover the underlying pathogenic mechanism of the disorder. For example, a number of genome-wide linkage studies have identified some main loci that are linked to MMD: 3p24.2-p26, 6q25, 8q23, 12p12, and 17q25. ${ }^{2}$ However, independent studies failed to identify the association between MMD and these loci, except for the $17 \mathrm{q} 25$ locus. Recent genetic studies have revealed that the RNF213 locus at $17 \mathrm{q} 25$ is an important susceptibility gene for MMD. ${ }^{6}$ However, there is still a lack of data on the precise role of this gene, and one report even suggests that this gene may not be solely responsible for MMD and that other susceptibility genes for MMD may exist. ${ }^{17}$

Accordingly, new approaches to and multimodal studies on the pathogenesis of MMD are required. In previous reports, endothelial progenitor cells (EPCs) or vascular progenitor cells were shown to play an important role in physiological or pathological angiogenesis. ${ }^{9}$ Abundant evidence suggests that circulating EPCs contribute to the development of diverse cardiovascular diseases. In particular, it has been documented that dysfunction in or reduced numbers of circulating EPCs is related to the pathogenesis of stroke. ${ }^{5}$ Interestingly, recent studies have shown an intimate correlation between circulating EPCs and the development of MMD. ${ }^{11,19}$ Moreover, it has also been reported that the circulating EPCs in MMD patients are dysfunctional; ${ }^{11}$ however, the reason for the dysfunction is not yet understood.

In this study, we investigated the cause of the dysfunction of circulating endothelial colony-forming cells (ECFCs), previously termed "EPCs," in MMD patients. We refer to EPCs as ECFCs because the outgrowth cells from adherent cultures of peripheral blood cells in vitro may have the different characteristics of pure EPCs. In the present study, we showed that the abnormalities in the mitochondria of ECFCs were related to the dysfunction of the circulating ECFCs in MMD patients.

As previously stated, MMD occurs more frequently among women. ${ }^{1,12}$ This female predominance is significantly more noticeable in the familial cases than in the sporadic cases. In familial cases, the ratio of women/ men significantly increases to 5.0. ${ }^{18}$ Moreover, it has been documented that the ratio of maternal transmission/paternal transmission increased more than 3-fold in familial MMD cases. ${ }^{16}$ We designed the present study according to these reports that MMD shows characteristic inheritance. This study is the first to analyze the mitochondria of ECFCs from MMD patients. With the results of our study, we present new insights into the pathogenesis of MMD.

\section{Methods \\ Study Participants}

We obtained blood samples from MMD patients during revascularization surgery after obtaining informed consent from the patients and/or their parents. All patients were confirmed to have MMD through angiographic study. The blood samples of 5 MMD patients ( 2 males and 3 females) were included in this study, and the median patient age was 13 years (range 2-36 years). For the control group, 5 healthy volunteers ( 2 men and 3 women) were recruited, and their median age was 23 years (range 20-27 years). These volunteers had no history of stroke, hypertension, or smoking. Clinical features of the MMD patients and control group are summarized in Table 1.

This study was approved by the institutional review board of Seoul National University Hospital. All participants gave written informed consent. A blind observer who did not know the clinical information for each patient performed a series of in vitro experiments to examine the ultrastructure and function of the mitochondria (variety of assays).

\section{Cell Culture and Phenotypic Characterization of the ECFCs}

All blood samples used for cultures were processed within 2 hours after collection. The peripheral blood was diluted 1:1 with phosphate-buffered saline (PBS), and the mononuclear cells were isolated from the buffy coat in Ficoll (1077 g/ml, Histopaque-1077, Sigma-Aldrich) and washed in PBS 3 times. The cells were plated on culture dishes coated with collagen Type I (BD BioCoat, BD Biosciences) and grown in an endothelial cell growth medium (EGM-2, Clonetics) at a density of $2 \times 10^{6}$ cells per well. The cells were maintained at $37^{\circ} \mathrm{C}$ and $5 \% \mathrm{CO}_{2}$ in a humidified atmosphere.

To verify that cells were ECFCs, we confirmed that they exhibited a typical cobblestone morphology and immunofluorescence staining by using antibodies against CD31, CD34, CD45, CD133, KDR, and vWF, as previously described in published studies. ${ }^{13}$

\section{RNF213 Gene Sequencing}

DNA samples were obtained from ECFCs using the DNA Mini kit (Invitrogen) according to the manufacturer's instructions. After DNA extraction and quantification, the DNA samples were subjected to PCR amplification with the appropriate primer sets (sense 5'-CTGATGCGTCAGCTCCATAG- $3^{\prime}$ and antisense 5'-TTCCTGCTTTGTGCAGTCAC-3'). Sequencing analysis of the RNF213 c. $14576 \mathrm{G}>\mathrm{A}$ variant was conducted on the ABI 3730XL DNA sequencer, and data were analyzed by $\mathrm{ABI}$ sequencing analysis software (Applied Biosystems).

\section{Electron Microscope Analysis}

The normal and MMD ECFCs were fixed overnight in a mixture of cold $2.5 \%$ glutaraldehyde in $0.1 \mathrm{M}$ of phosphate buffer ( $\mathrm{pH} 7.2)$ and $2 \%$ paraformaldehyde in $0.1 \mathrm{M}$ of phosphate or cacodylate buffer ( $\mathrm{pH}$ 7.2) and then embedded with epoxy resin. The epoxy resin-mixed samples were loaded into capsules and allowed to polymerize at $38^{\circ} \mathrm{C}$ for 12 hours and $60^{\circ} \mathrm{C}$ for 48 hours. Thin sections were sliced on an ultramicrotome (RMC MT-XL) and collected on a copper grid. Appropriate areas were cut into thin sections at $65 \mathrm{~nm}$ and stained with saturated $4 \%$ uranyl acetate and $4 \%$ lead citrate solutions, followed by examination under a transmission electron microscope (JEM-1400, Jeol Ltd.) at $80 \mathrm{kV}$.

\section{Quantitative Morphological Analysis of the Mitochondria}

The mitochondria were visualized after the cells were 
TABLE 1. Clinical features of the MMD patients and healthy volunteers

\begin{tabular}{|c|c|c|c|c|c|c|c|}
\hline $\begin{array}{l}\text { Case } \\
\text { No. }\end{array}$ & Sex & $\begin{array}{l}\text { Age } \\
\text { (yrs) }\end{array}$ & $\begin{array}{c}\text { RNF213 c. } 14576 \mathrm{G}>\mathrm{A} \\
\text { Variant }\end{array}$ & Symptoms & MRI Findings & Angiographic Findings & $\begin{array}{l}\text { Suzuki } \\
\text { Grade }\end{array}$ \\
\hline MMD 1 & $\mathrm{~F}$ & 36 & $\mathrm{G} / \mathrm{A}$ & Headache, TIA & Ischemic lesions in the WM & Bilat, no PCA involvement & IV/IV \\
\hline MMD 2 & M & 8 & $\mathrm{G} / \mathrm{A}$ & TIA & No infarct & Bilat, no PCA involvement & $\| I I / I I \mid$ \\
\hline MMD 3 & $\mathrm{~F}$ & 4 & $\mathrm{G} / \mathrm{A}$ & Headache, TIA & Multiple old lacunar infarcts & Bilat, no PCA involvement & $\| I I / I I \mid$ \\
\hline MMD 4 & $\mathrm{~F}$ & 17 & $\mathrm{~A} / \mathrm{A}$ & TIA & No infarct & Bilat, no PCA involvement & $\|I I /\|$ \\
\hline MMD 5 & M & 1.5 & $\mathrm{~A} / \mathrm{A}$ & TIA & No infarct & Bilat, no PCA involvement & $\|/\|$ \\
\hline Control 1 & $\mathrm{~F}$ & 27 & $\mathrm{G} / \mathrm{G}$ & None & - & - & - \\
\hline Control 2 & M & 23 & G/G & None & - & - & - \\
\hline Control 3 & $\mathrm{~F}$ & 23 & G/G & None & - & - & - \\
\hline Control 4 & $M$ & 20 & $\mathrm{G} / \mathrm{A}$ & None & - & - & - \\
\hline Control 5 & $\mathrm{~F}$ & 24 & $\mathrm{G} / \mathrm{G}$ & None & - & - & - \\
\hline
\end{tabular}

$\mathrm{A} / \mathrm{A}=$ homozygote (genotype AA); G/A = heterozygote (genotype GA); G/G = wild type (genotype GG); PCA = posterior cerebral artery; TIA = transient ischemic attack; WM $=$ white matter; $-=$ not applicable.

None of the patients or volunteers had associated conditions including neurofibromatosis type 1, Down syndrome, previous cranial irradiation, diabetes mellitus, hypertension, high cholesterol level, excessive alcohol intake, carotid or other artery disease, and heart disease. Neither did any of the patients or volunteers have a family history of stroke.

stained with MitoTracker Red dye (Invitrogen). Images were captured under a confocal laser scanning microscope (FV10i-w, Olympus), and 20-50 cells from each group were analyzed with ImageJ software (National Institutes of Health), as previously described. ${ }^{3}$

\section{Measurements of the Oxygen Consumption Rate}

An extracellular flux analyzer (XF24-3, Seahorse Bioscience) was used to measure the oxygen consumption rate (OCR) in the medium immediately surrounding the ECFCs cultured in XF24 V7 cell culture microplates (Seahorse Bioscience), according to the manufacturer's instructions. Briefly, the OCR measurement was performed after equilibration in assay medium (lacking supplemental $\mathrm{CO}_{2}$ ). The Seahorse analyzer uses a cartridge with 24 optical fluorescent $\mathrm{O}_{2}$ sensors that are embedded in a sterile disposable cartridge, one for each well. Prior to each rate measurement, the plungers mix the assay medium in each well for 8 minutes to allow the partial $\mathrm{O}_{2}$ pressure to reach equilibrium. To measure the rates, the plungers gently descend into the wells to form chambers. The $\mathrm{O}_{2}$ concentration is periodically measured over 4 minutes, and the rates of $\mathrm{O}_{2}$ consumption are obtained from the slopes of the changes in the concentration versus time. After the rates are measured, the plungers ascend, and the wells are gently mixed to equilibrate the medium. The baseline rates are measured 4 times. One or more test chemicals are preloaded in the reagent delivery chambers of the sensor cartridge and then pneumatically injected into the wells to reach the desired final working concentration. After 2 minutes of mixing, the postexposure OCR was measured 4-6 times. The averages of 4 baseline rates and 2-6 test rates were used for the data analyses.

\section{Mitochondrial Membrane Potential Measurement}

The mitochondrial membrane potential (MMP) measurements were performed as previously described. ${ }^{20} \mathrm{In}$ depolarized cells, labeling of the mitochondria with potential-indicating probes such as tetramethylrhodamine methyl ester (TMRM) disappears; therefore, the red fluorescence serves as an indicator of the MMP. The medium was replaced with phenol red-free medium containing $500 \mathrm{nM}$ TMRM (100 $\mu \mathrm{l} /$ well, T-668, Invitrogen). The plates were incubated for 1 hour at $37^{\circ} \mathrm{C}$ and washed 3 times with PBS (50 $\mu \mathrm{l} / \mathrm{well})$. The fluorescent signals were captured using a fluorescence microscope (Olympus), and $>100$ cells from each group (MMD patients and healthy volunteers) were analyzed.

\section{Fluorescence Imaging to Measure the Intracellular $\mathrm{Ca}^{2+}$ Concentrations}

To image the intracellular $\mathrm{Ca}^{2+}$ concentrations $\left(\left[\mathrm{Ca}^{2+}\right]_{\mathrm{i}}\right)$, the cells were loaded with the $\mathrm{Ca}^{2+}$-sensitive dye fluo-4 acetoxymethyl ester (Fluo-4; $5 \mu \mathrm{M}, \mathrm{F} 10471$, Invitrogen) for 60 minutes at $37^{\circ} \mathrm{C}$ and then washed with PBS to remove the extracellular Fluo-4. The fluorescent signals were captured using a fluorescence microscope. The fluorescence intensity reflected $\left[\mathrm{Ca}^{2+}\right]_{\mathrm{i}}$. The images for 100 cells from each group (MMD patients and healthy volunteers) were analyzed using ImageJ software.

\section{MTT Assay}

To evaluate the activities of mitochondrial enzymes, MTT [3-(4,5-dimethylthiazol-2-yl)-2,5-diphenyltetrazolium bromide)] assays, which measure mitochondrial dehydrogenase activity, were performed as previously described. ${ }^{4}$ Briefly, $2.5 \mathrm{mg} / \mathrm{ml}$ of MTT (M2003, SigmaAldrich) in phenol red-free medium was added to the cells and incubated for 2 hours at $37^{\circ} \mathrm{C}$. Then, the MTT solution was aspirated, isopropanol was added to dissolve the formazan crystals, and the cells were incubated for 1 hour at $37^{\circ} \mathrm{C}$. The absorbance was measured at $540 \mathrm{~nm}$. At least 3 independent experiments were performed, and the data were expressed as a percentage of the normal ECFCs. 

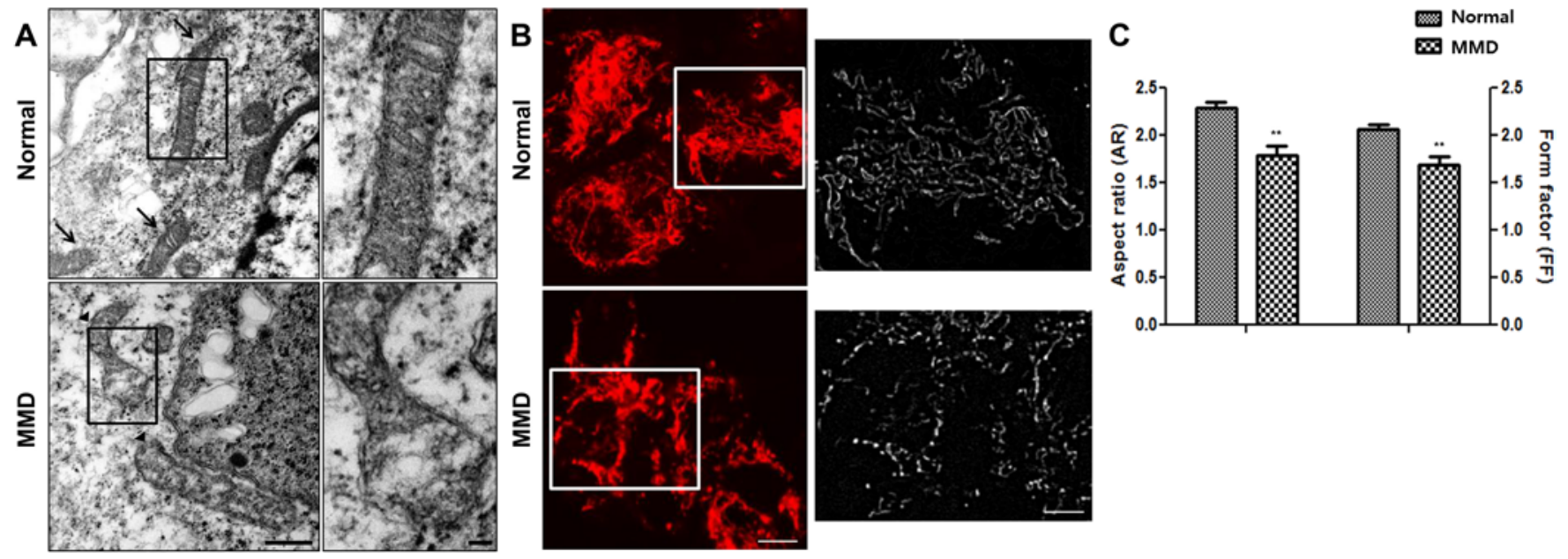

FIG. 1. A: Electron microscopy reveals disrupted mitochondrial morphology in the MMD ECFCs. Arrows indicate the intact and healthy mitochondria, whereas arrowheads indicate the disrupted mitochondria. Insets in the left images are magnified on the right. Bars $=0.5$ and $0.1 \mu \mathrm{m}$, respectively. B: Changes in the mitochondrial morphology in the MMD ECFCs are shown by MitoTracker staining. Insets in the left images are magnified on the right. The enlarged images were converted to an 8-bit format using the ImageJ program to analyze mitochondrial morphology. Bars $=5$ and $2 \mu \mathrm{m}$, respectively. C: Quantitative analysis of changes in mitochondrial morphology. Mitochondria in the MMD ECFCs were shorter (represented by the aspect ratio) and had a more circular shape (represented by the form factor) than mitochondria in the normal ECFCs. ${ }^{* *} p<0.01$, compared with normal ECFCs.

\section{Measurement of Reactive Oxygen Species Levels in the ECFCs}

To detect the intracellular reactive oxygen species (ROS) levels, we used 2',7'-dichlorodihydrofluorescein diacetate (DCFH-DA; Molecular Probes). Approximately $10^{6}$ cells were incubated with DCFH-DA $(10 \mu \mathrm{M})$ for 30 minutes at $37^{\circ} \mathrm{C}$ in the dark, and the cells were washed twice with PBS. Fluorescence was detected with a FACScan flow cytometer (Becton Dickinson) using CellQuest software (Becton Dickinson).

\section{Capillary Tube Formation Assay}

Functional activity of the ECFCs was evaluated using an in vitro capillary tube formation assay. The ECFCs $(2 x$ $10^{4}$ cells/well) were plated on a Matrigel-coated (BD Biosciences) 48-well plate and incubated for 18 hours. The number of tubes and branches attached to tubular structures that had formed from the ECFCs were counted in 4 randomly selected microscope fields (original magnification $\times 40$ ).

\section{Data Analysis}

All data from at least 3 independent experiments for each data point were expressed as the mean \pm standard error of the mean. The Student t-test was used for 2-group comparisons, and an ANOVA followed by Fisher's least significant difference (LSD) post hoc test was used to compare 3 or more groups using SigmaStat for Windows version 3.10 (Systat Software Inc.).

\section{Results}

\section{Disrupted Mitochondrial Morphology in MMD ECFCs}

To investigate the mechanism of impaired function in the MMD ECFCs, we focused on the mitochondria be- cause previous studies showed that mitochondrial dysfunction leads to alterations in cellular function and ultimately cell death. Using electron microscopy analysis, massive mitochondrial fission and loss of cristae were observed in the MMD ECFCs but not in the normal ECFCs (Fig. 1A). Consistent with the electron microscopy data, mitochondria in the MMD ECFCs were shorter (represented by the aspect ratio) and more circular (represented by the form factor) than those in the normal ECFCs (Fig. 1B and C). These data indicate that the MMD ECFCs displayed an altered mitochondrial morphology compared with that in the normal ECFCs.

\section{Mitochondrial Dysfunction and Increased $\left[\mathrm{Ca}^{2+}\right]_{i}$ in MMD ECFCs}

We determined whether the MMD ECFCs exhibited mitochondrial dysfunction as well as altered mitochondrial morphology. To investigate any alterations in mitochondrial function in the normal and MMD ECFCs, we first evaluated cellular respiration in the normal and MMD ECFCs by measuring the OCR. To measure adenosine triphosphate (ATP) production, oligomycin was injected into culture wells before injection of the uncoupling agent carbonyl cyanide m-chlorophenyl hydrazone (CCCP). The MMD ECFCs consistently showed reduced basal OCRs, OCRs coupled to ATP generation, which is the oligomycin-sensitive OCR, and total oxidative capacity, which is the CCCP-sensitive OCR (Fig. 2A). The basal respiration rates, ATP levels, and oxidative capacity of the MMD ECFCs were decreased by approximately $70 \%, 80 \%$, and $75 \%$ relative to the normal ECFCs, respectively (Fig. 2B). The antimycin-insensitive OCR, which is interpreted as nonmitochondrial respiration, was slightly but nonsignificantly reduced in the MMD ECFCs.

Next, we showed that the MMP in the MMD ECFCs 
A
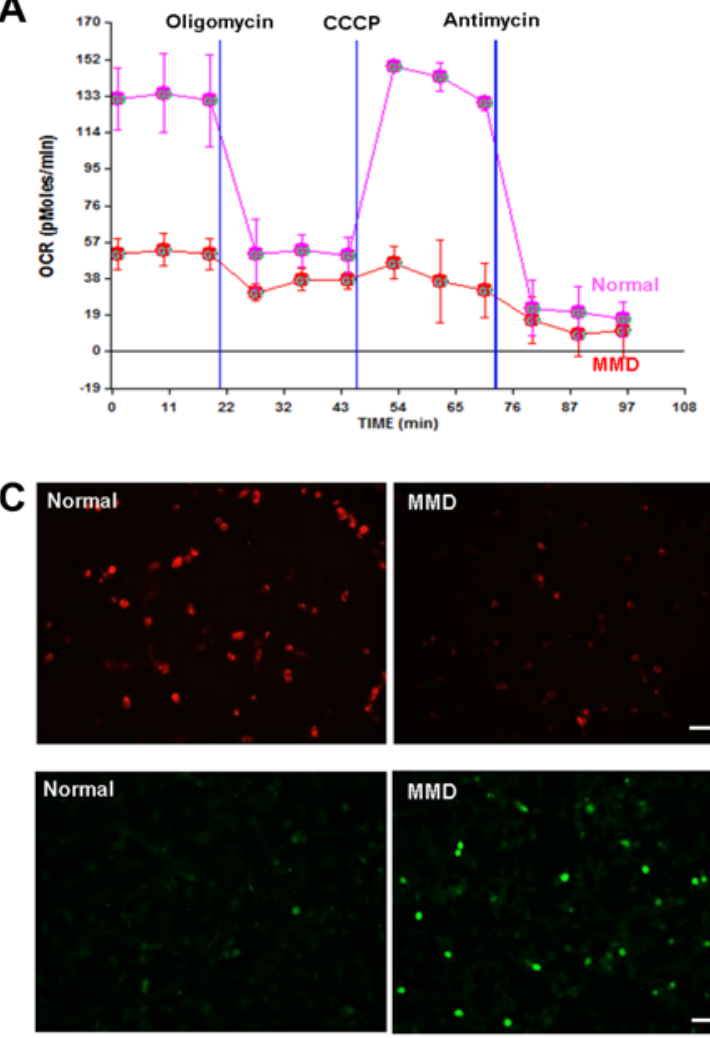
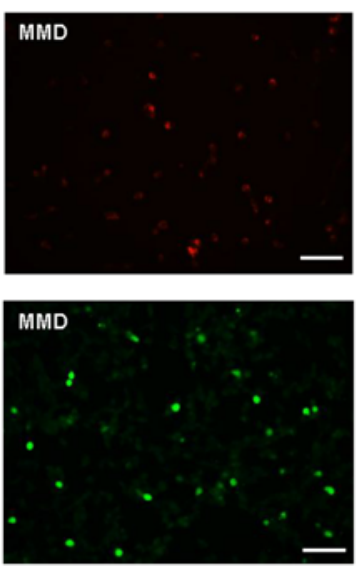

B
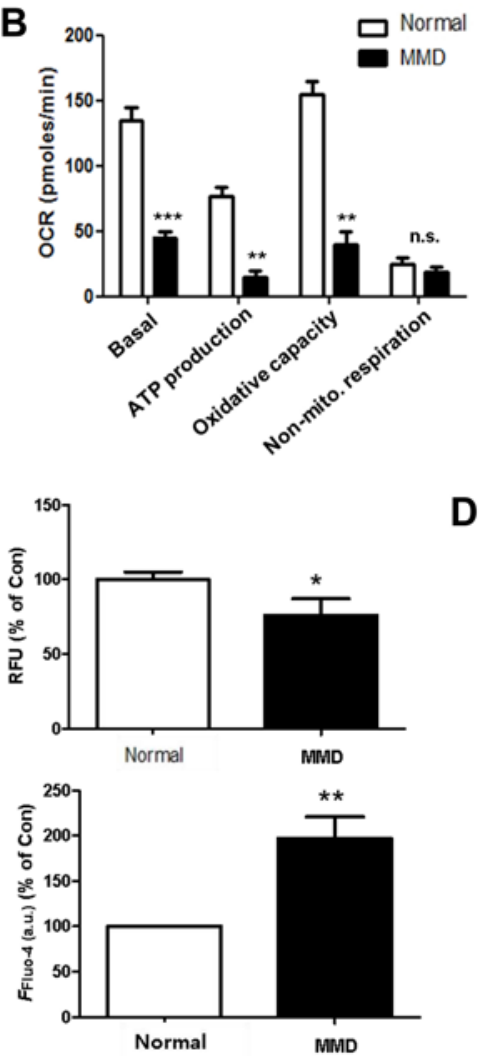
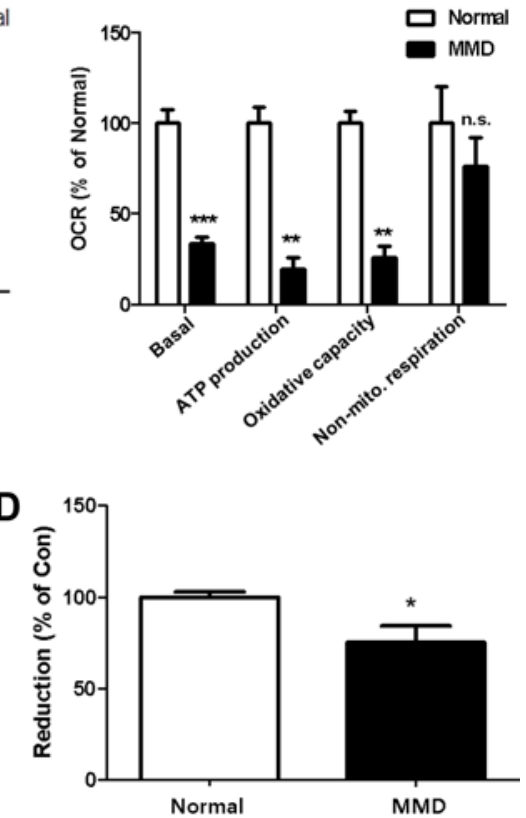

FIG. 2. The ECFCs from patients with MMD displayed mitochondrial dysfunction and increased $\left[\mathrm{Ca}^{2+}\right]_{\text {. }} \mathrm{A}$ : Representative data from a single Seahorse experiment that was representative of 3 independent experiments depict the OCR in normal (pink) and MMD (red) ECFCs. Each OCR data point represents a mean \pm standard error the mean. The concentrations of oligomycin (complex $V$ inhibitor), CCCP (uncoupler of mitochondrial respiration and ATP synthesis), and antimycin (complex III inhibitor) were 2, 5, and $1 \mu \mathrm{M}$, respectively. The MMD ECFCs displayed reduced OCR levels. B: Graphs show total OCR (left) as well as the fractions of OCR (right) that was changed after the administration of oligomycin, CCCP, and antimycin. Data were derived from the experiment represented in panel $A .{ }^{* *} p<0.01$ and ${ }^{* *} p<0.001$, both compared with normal ECFCs. C: The MMP was measured using the TMRM assay (upper). Intracellular $\mathrm{Ca}^{2+}$ was measured using the Fluo-4 assay (lower). Representative images (left) are shown, and bar graphs (right) represent data expressed as the means \pm standard error of the mean. ${ }^{*} p<0.05$ and ${ }^{* *} p<0.01$, both compared with normal ECFCs. Bar $=50 \mu \mathrm{m}$. D: Mitochondrial dehydrogenase activity was determined by the MTT assay. ${ }^{*} p<$ 0.05, compared with normal ECFCs. a.u. = arbitrary units; FFlou-4 = fluorescence Fluo-4; Non-mito. = nonmitochondrial; n.s. = not significant; $\%$ of $\mathrm{Con}=\%$ of concentration; RFU $=$ relative fluorescence unit.

was significantly attenuated by using TMRM assays (Fig. $2 \mathrm{C}$ ). It is known that mitochondria are an important $\mathrm{Ca}^{2+}$ buffering organelle that sustains cellular $\mathrm{Ca}^{2+}$ hemostasis and that $\left[\mathrm{Ca}^{2+}\right]_{\mathrm{i}}$ accumulation induces cell death. ${ }^{20} \mathrm{Be}-$ cause the MMD ECFCs exhibited mitochondrial dysfunction, we analyzed the $\left[\mathrm{Ca}^{2+}\right]_{\mathrm{i}}$ levels in ECFCs by using a Fluo-4 assay. We found that the $\left[\mathrm{Ca}^{2+}\right]_{\mathrm{i}}$ levels in the MMD ECFCs were increased. Furthermore, the MTT assay was performed to investigate the activity of the mitochondrial enzymes in the ECFCs. We found that the MMD ECFCs exhibited significantly reduced mitochondrial reductase activity (Fig. 2D). Overall, these data indicate that the MMD ECFCs, as compared with the normal ECFCs, exhibited mitochondrial dysfunction.

\section{Increased ROS Levels in MMD ECFCs}

Because a depolarized MMP is known to induce ROS generation $^{22}$ and the intracellular ROS levels are inextricably linked to mitochondrial function, we measured the intracellular ROS levels in the ECFCs from the MMD patients and normal controls. The ROS levels were significantly increased in the MMD ECFCs compared with levels in the normal ECFCs (132\% of normal control, $p=$ 0.041; Fig. 3A).

Furthermore, we treated the MMD ECFCs with the ROS scavenger $N$-acetyl-L-cysteine (NAC). This treatment dose-dependently reduced the intracellular ROS production in the MMD ECFCs ( $p=0.006$; Fig. 3B and C)

\section{Mitochondrial Abnormalities Reversed by the ROS Scavenger Treatment}

The MMD ECFCs were treated with NAC to determine whether the ROS scavenger could reverse the observed mitochondrial abnormalities. Surprisingly, the NAC-treated MMD ECFCs displayed healthy mitochondria in terms of their length and shape (Fig. 4A). When the OCR was analyzed in the ECFCs after a 24-hour treatment with NAC, we found that the NAC treatment significantly restored the 

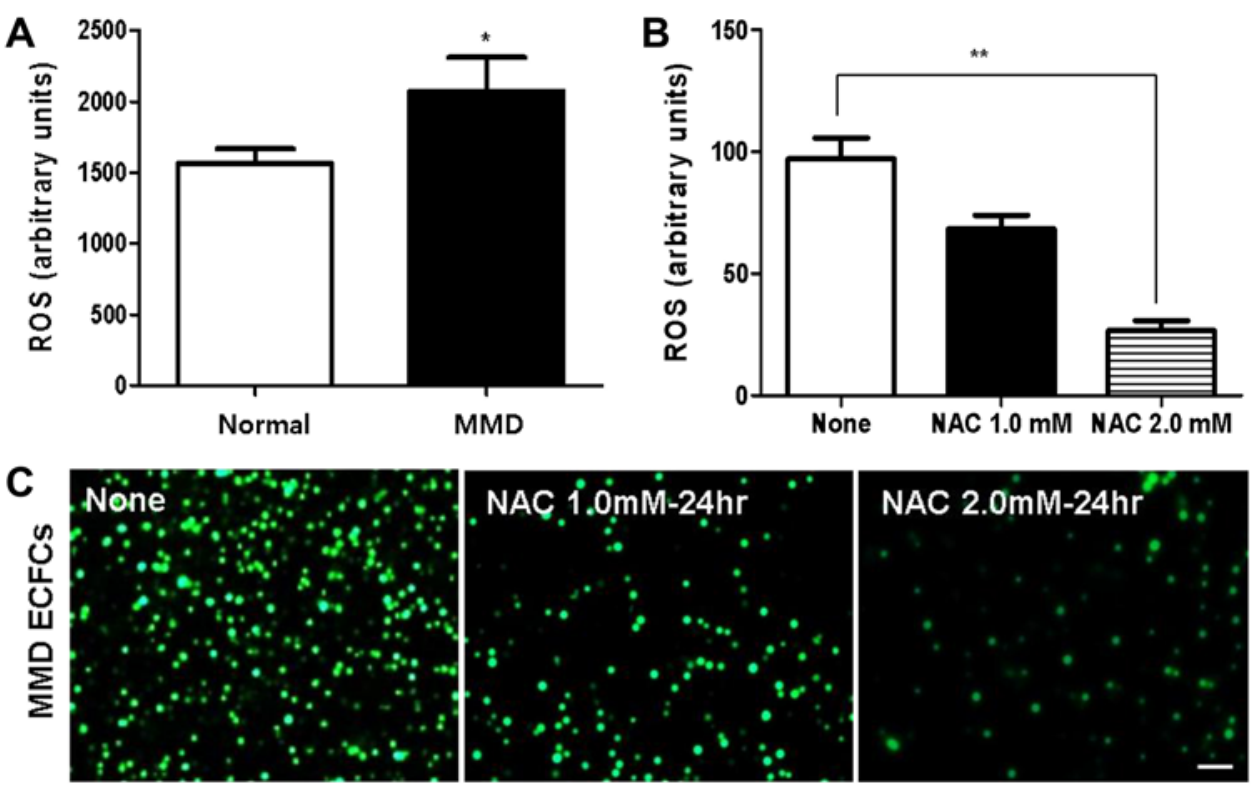

FIG. 3. A: The ROS levels in ECFCs were measured using the oxidation-dependent fluorescent dye DCFH-DA. Generation of ROS levels was significantly increased in the MMD ECFCs. ${ }^{*} p<0.05$, compared with normal ECFCs. B: The NAC treatment dose-dependently reduced the ROS levels in the MMD ECFCs. ${ }^{* *} p<0.01$, compared with normal ECFCs. C: Representative images showing fluorescing ROS without (None) and with (NAC $1.0 \mathrm{mM}$ and $2.0 \mathrm{mM}$ ) NAC treatment.

basal respiration rates, ATP levels, and oxidative capacity in the MMD ECFCs (Fig. 4B). Using the TMRM assay, we showed that the MMP was also restored in the NAC-treated MMD ECFCs (Fig. 4C). In addition, the Fluo-4 assay showed that the NAC-treated MMD ECFCs maintained normal $\left[\mathrm{Ca}^{2+}\right]_{\mathrm{i}}$. These data indicated that the increased ROS levels induced harmful effects on the mitochondria in the MMD ECFCs and that treatment with an ROS scavenger could reverse the mitochondrial dysfunction in the MMD ECFCs.

\section{Restoration of Impaired Angiogenic Activity in MMD ECFCs via ROS Scavenger Treatment}

Tube formation is an important function of ECFCs, and in vitro tube formation tests are the most frequently used assays for measuring angiogenic activity. We checked whether treatment with the ROS scavenger restored the impaired angiogenic activity in the MMD ECFCs. Interestingly, the NAC treatment $(2 \mathrm{mM})$ significantly increased the number of tubes formed and even enhanced the angiogenic activity (Fig. 5).

\section{Discussion}

The current study indicates that the mitochondria of ECFCs from MMD patients exhibit morphological and functional abnormalities. The morphology of the mitochondria in the ECFCs from MMD patients was fragmented with vacuoles and disrupted cristae, whereas that in normal ECFCs appeared to be elongated and filamentous. Functionally, the mitochondria in ECFCs from MMD patients exhibited reduced basal respiration, ATP production, and total oxidative capacity (maximal respiration), which indicates that the ECFCs from MMD patients are functionally defective. Furthermore, the mitochondria in the ECFCs from MMD patients were more depolarized and exhibited increased ROS and $\left[\mathrm{Ca}^{2+}\right]_{\mathrm{i}}$ levels and reduced mitochondrial reductase activity. Notably, a scavenger that only decreased the ROS levels not only reversed the morphological and functional abnormalities of the mitochondria but also restored the angiogenic activity of the ECFCs. Accordingly, the mitochondria may have a significant role in the pathogenesis of MMD.

Mitochondria are involved in energy production as well as other cellular activities, such as signaling cascades, cellular proliferation, senescence, and cell death. ${ }^{15}$ Thus, cellular dysfunction is highly associated with abnormalities in mitochondrial status and activity. Actually, mitochondrial dysfunction results in numerous diseases, including neurodegenerative disorders, metabolic diseases, and even cancers that are known as "mitochondrial diseases" or "mitochondrial disorders." 10

Numerous studies have demonstrated that mitochondrial damage and dysfunction have an important role in various cardiovascular disorders, such as atherosclerosis, hypertension, stroke, heart failure, and ischemic heart disease. ${ }^{23}$ However, there has been no report about the correlation between mitochondria and MMD, until now. Our study indicates that the impaired function of the ECFCs from MMD patients may result from mitochondrial abnormalities, suggesting that MMD may be a mitochondria-related disease. To prove this hypothesis, however, mutations in the nuclear DNA that encode mitochondrial components or mutations in the mitochondrial DNA should be identified. Such investigations are underway. Additional studies of the diverse factors that contribute to the mitochondrial abnormalities of the ECFCs in MMD patients are required. Such studies could clearly identify mitochondrial abnor- 

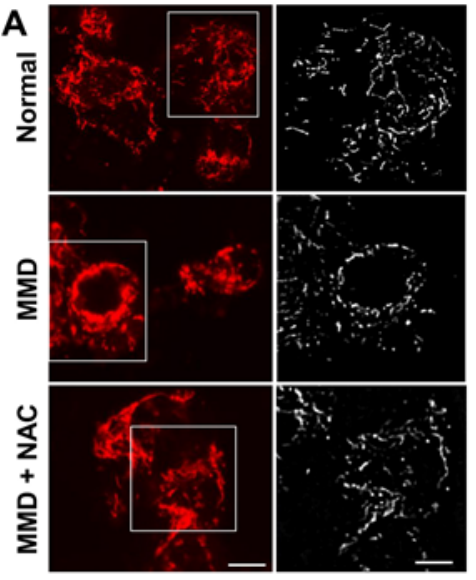

B
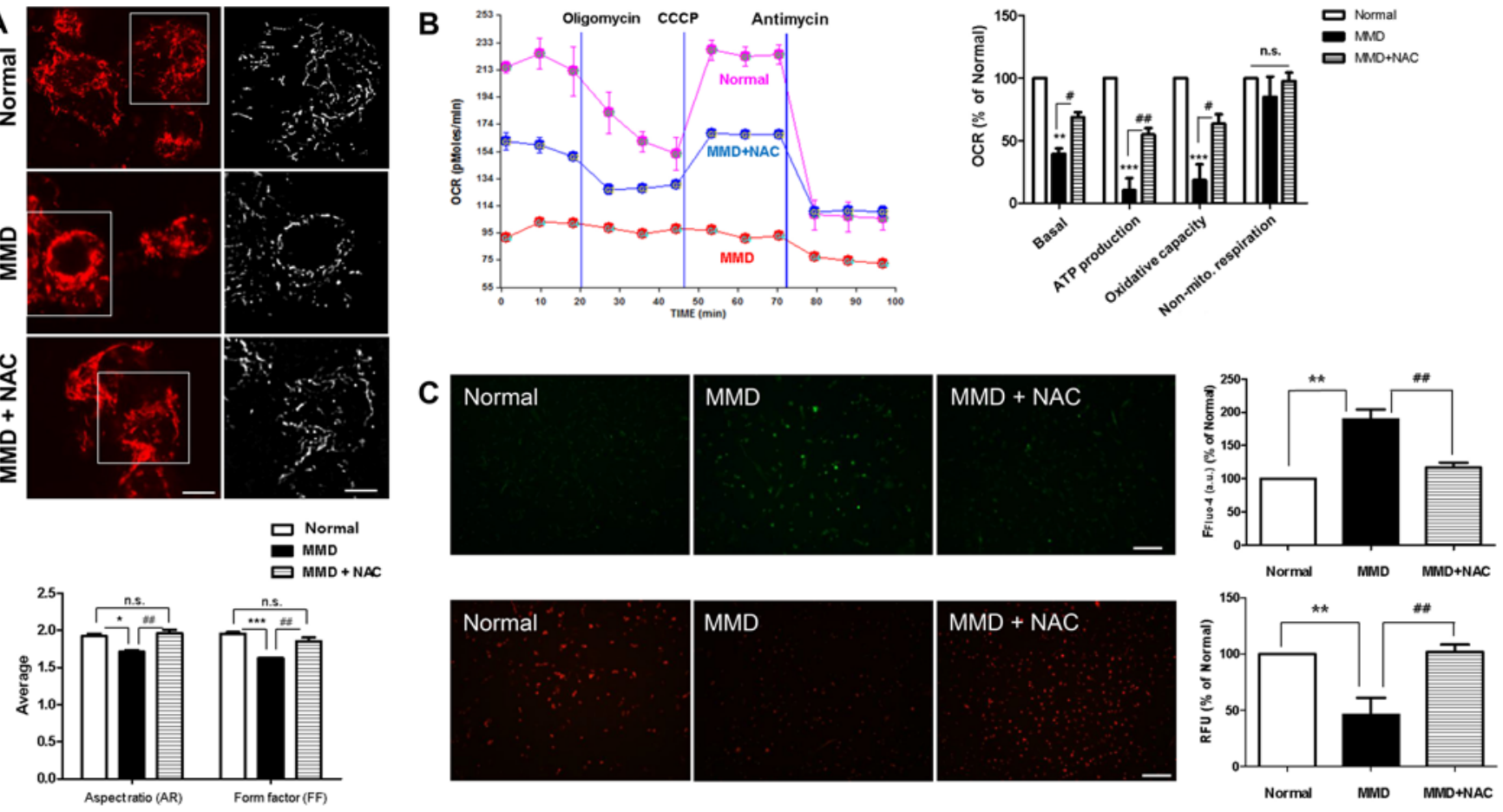

FIG. 4. A: Treatment with the ROS scavenger NAC reversed the mitochondrial abnormalities in the MMD ECFCs (upper). Insets in the left images are magnified on the right. The enlarged images were converted to an 8-bit format using the ImageJ program to analyze mitochondrial morphology. Bar graph (lower) representing quantitative analysis of the mitochondrial morphology after the ROS scavenger treatment. ${ }^{*} p<0.05$ and ${ }^{* * *} p<0.001$, both compared with normal ECFCs; \#\#p $<0.01$, compared with MMD ECFCs (30 samples each). Bars $=5$ and $2 \mu \mathrm{m}$, respectively. B: Representative data (left) from a single Seahorse experiment that was representative of 3 independent experiments depict the OCR in normal (pink), MMD (red), and NAC-treated MMD (purple) ECFCs. Each OCR data point represents a mean \pm standard error of the mean. Bar graph (right) shows fractions of OCR that were changed after the administration of oligomycin, CCCP, and antimycin, whose concentrations were 2,5 , and $1 \mu \mathrm{M}$, respectively. ${ }^{* *} p<0.01$ and ${ }^{* * *} p<0.001$, both compared with normal ECFCs; \#p $<0.05$ and \#\#p < 0.01, both compared with MMD ECFCs. C: Representative images (left) are shown, and bar graphs (right) represent data expressed as the mean \pm standard error of the mean. The MMP was measured using the TMRM assay (lower). ${ }^{* *} p<0.05$, compared with normal ECFCs; \#\#p $<0.01$, compared with MMD ECFCs. Intracellular Ca ${ }^{2+}$ was measured using the Fluo-4 assay (upper). ${ }^{* *} p<0.01$, compared with normal ECFCs; \#\#p $<0.01$, compared with MMD ECFCs. Bar $=50 \mu \mathrm{m}$.

malities in MMD as the primary phenomenon rather than a secondary result.

There is increasing evidence that circulating ECFCs, which were previously called "EPCs," have a very important role in endothelial homeostasis. For example, it is well documented that circulating ECFCs contribute to ongoing endothelial repair? ${ }^{7}$ Moreover, ECFC dysfunction plays an important role in the pathogenesis of MMD. Although there are still controversial results and the exact role of ECFCs has not been conclusively defined, many studies have demonstrated an intimate connection between ECFCs and MMD. ${ }^{11,21}$

In previous studies, we demonstrated not only that there were fewer ECFCs in MMD patients but that the ECFCs also showed impaired differentiation, dysfunction, and a tendency to undergo senescence. ${ }^{8,13}$ The current study indicates that these impaired functions and the senescence of ECFCs in MMD patients might result from mitochondrial abnormalities. These abnormalities may be related to the delayed repair of the damaged vessel and the development of vessel occlusions in MMD patients.
According to our data in this study, mitochondrial dysfunction may be an important causative risk factor for the defects in ECFCs in MMD patients. This result implies that MMD may be a mitochondria-related disease. Furthermore, our study showed that ROS were closely related to the function of ECFCs. This finding provides a clue to the pathological mechanism of and the therapeutic targets for MMD, such as ROS-sensitive transcription factors. Recently, a study demonstrated that microRNA-424 is closely related to the cerebral ischemia-reperfusion injury in ischemic stroke by regulating endogenous ROS. ${ }^{14}$ We assume that ROS plays an important role in the pathogenesis of MMD, and we have an ongoing study into this issue.

There are several limitations to our study. Firstly, the sample size is relatively small. Our findings should be verified in a larger cohort. Moreover, we could not recruit age-matched controls. However, it is ethically undesirable to obtain blood from healthy pediatric volunteers, because children are vulnerable subjects in the medical research field. Lastly, given the practical difficulty of acquiring the "pathological" tissue of MMD patients (the intracranial 
A

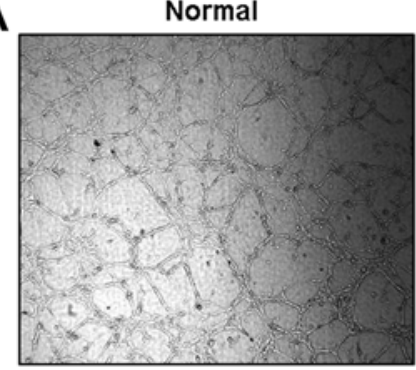

B

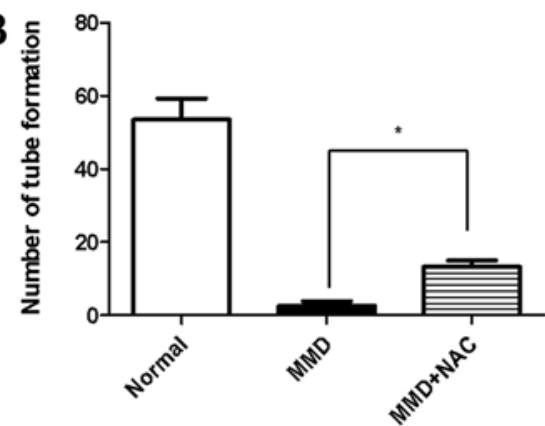

MMD

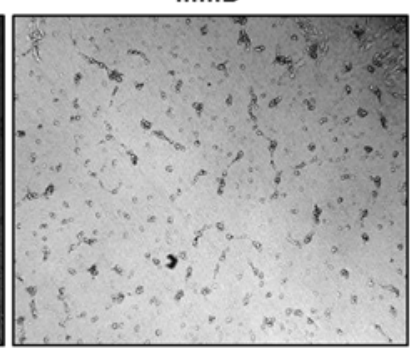

MMD + NAC

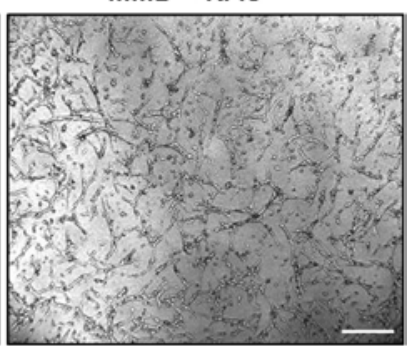

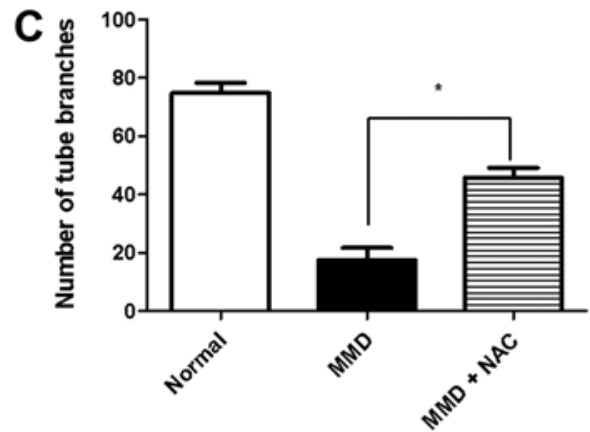

FIG. 5. Treatment with the ROS scavenger restored the impaired angiogenic activity in the ECFCs from patients with MMD. A: Tube formation assay using ECFCs cultivated on Matrigel. B: The number of tubes formed by the NAC-treated MMD ECFCs was significantly increased. ${ }^{*} p<0.01$, compared with MMD ECFCs. C: The number of tube branches was also significantly increased in the NAC-treated MMD ECFCs. ${ }^{*} p<0.01$, compared with MMD ECFCs.

vessels such as moyamoya vessels or the stenotic carotid artery), ECFCs were used for the study.

\section{Conclusions}

Our study revealed morphological and functional abnormalities of the mitochondria in ECFCs from MMD patients. This finding suggests that MMD may be a mitochondria-related disease. Additional studies on mitochondria could contribute new insights into the pathogenesis of MMD.

\section{Acknowledgments}

This research was supported by a grant (No. HI12C0066) from the Korea Health Technology R\&D Project through the Korea Health Industry Development Institute (KHIDI), funded by the Ministry of Health \& Welfare, Republic of Korea.

\section{References}

1. Baba T, Houkin K, Kuroda S: Novel epidemiological features of moyamoya disease. J Neurol Neurosurg Psychiatry 79:900-904, 2008

2. Bersano A, Guey S, Bedini G, Nava S, Hervé D, Vajkoczy P, et al: Research progresses in understanding the pathophysiology of moyamoya disease. Cerebrovasc Dis 41:105-118, 2016

3. Byun J, Son SM, Cha MY, Shong M, Hwang YJ, Kim Y, et al: CR6-interacting factor 1 is a key regulator in $\mathrm{A} \beta$-induced mitochondrial disruption and pathogenesis of Alzheimer's disease. Cell Death Differ 22:959-973, 2015

4. Cha MY, Han SH, Son SM, Hong HS, Choi YJ, Byun J, et al: Mitochondria-specific accumulation of amyloid $\beta$ induces mitochondrial dysfunction leading to apoptotic cell death. PLoS One 7:e34929, 2012
5. Chu K, Jung KH, Lee ST, Park HK, Sinn DI, Kim JM, et al: Circulating endothelial progenitor cells as a new marker of endothelial dysfunction or repair in acute stroke. Stroke 39:1441-1447, 2008

6. Fujimura M, Sonobe S, Nishijima Y, Niizuma K, Sakata H, Kure S, et al: Genetics and biomarkers of moyamoya disease: significance of RNF213 as a susceptibility gene. J Stroke 16: $65-72,2014$

7. Hill JM, Zalos G, Halcox JP, Schenke WH, Waclawiw MA, Quyyumi AA, et al: Circulating endothelial progenitor cells, vascular function, and cardiovascular risk. N Engl J Med 348:593-600, 2003

8. Kang HS, Moon YJ, Kim YY, Park WY, Park AK, Wang KC, et al: Smooth-muscle progenitor cells isolated from patients with moyamoya disease: novel experimental cell model. J Neurosurg 120:415-425, 2014

9. Kang HS, Wang KC, Kim SK: Circulating vascular progenitor cells in moyamoya disease. J Korean Neurosurg Soc 57:428-431, 2015

10. Khan NA, Govindaraj P, Meena AK, Thangaraj K: Mitochondrial disorders: challenges in diagnosis \& treatment. Indian J Med Res 141:13-26, 2015

11. Kim JH, Jung JH, Phi JH, Kang HS, Kim JE, Chae JH, et al: Decreased level and defective function of circulating endothelial progenitor cells in children with moyamoya disease. J Neurosci Res 88:510-518, 2010

12. Kuriyama S, Kusaka Y, Fujimura M, Wakai K, Tamakoshi A, Hashimoto S, et al: Prevalence and clinicoepidemiological features of moyamoya disease in Japan: findings from a nationwide epidemiological survey. Stroke 39:42-47, 2008

13. Lee JY, Moon YJ, Lee HO, Park AK, Choi SA, Wang KC, et al: Deregulation of retinaldehyde dehydrogenase 2 leads to defective angiogenic function of endothelial colony-forming cells in pediatric moyamoya disease. Arterioscler Thromb Vasc Biol 35:1670-1677, 2015

14. Liu P, Zhao H, Wang R, Wang P, Tao Z, Gao L, et al: MicroRNA-424 protects against focal cerebral ischemia and 
reperfusion injury in mice by suppressing oxidative stress. Stroke 46:513-519, 2015

15. McBride HM, Neuspiel M, Wasiak S: Mitochondria: more than just a powerhouse. Curr Biol 16:R551-R560, 2006

16. Mineharu Y, Takenaka K, Yamakawa H, Inoue K, Ikeda H, Kikuta KI, et al: Inheritance pattern of familial moyamoya disease: autosomal dominant mode and genomic imprinting. J Neurol Neurosurg Psychiatry 77:1025-1029, 2006

17. Moteki Y, Onda H, Kasuya H, Yoneyama T, Okada Y, Hirota $\mathrm{K}$, et al: Systematic validation of RNF213 coding variants in Japanese patients with moyamoya disease. J Am Heart Assoc 4:e001862, 2015

18. Nanba R, Kuroda S, Tada M, Ishikawa T, Houkin K, Iwasaki Y: Clinical features of familial moyamoya disease. Childs Nerv Syst 22:258-262, 2006

19. Rafat N, Beck GCh, Peña-Tapia PG, Schmiedek P, Vajkoczy $P$ : Increased levels of circulating endothelial progenitor cells in patients with moyamoya disease. Stroke 40:432-438, 2009

20. Son SM, Byun J, Roh SE, Kim SJ, Mook-Jung I: Reduced IRE1 $\alpha$ mediates apoptotic cell death by disrupting calcium homeostasis via the InsP3 receptor. Cell Death Dis 5:e1188, 2014

21. Sugiyama T, Kuroda S, Nakayama N, Tanaka S, Houkin K: Bone marrow-derived endothelial progenitor cells participate in the initiation of moyamoya disease. Neurol Med Chir (Tokyo) 51:767-773, 2011

22. Suski JM, Lebiedzinska M, Bonora M, Pinton P, Duszynski J, Wieckowski MR: Relation between mitochondrial membrane potential and ROS formation. Methods Mol Biol 810:183205, 2012

23. Yu E, Mercer J, Bennett M: Mitochondria in vascular disease. Cardiovasc Res 95:173-182, 2012

\section{Disclosures}

The authors report no conflict of interest concerning the materials or methods used in this study or the findings specified in this paper.

\section{Author Contributions}

Conception and design: Kim, JW Choi, Mook-Jung, Wang. Acquisition of data: JW Choi, Son, Moon, Byun. Analysis and interpretation of data: Son, Mook-Jung, Moon. Drafting the article: JW Choi, Son, Moon. Critically revising the article: Kim, Lee, Wang, Kang, Phi. Reviewed submitted version of manuscript: MookJung, Lee, Wang, Kang, Phi, SA Choi, Chong, Byun. Approved the final version of the manuscript on behalf of all authors: Kim. Administrative/technical/material support: SA Choi, Chong. Study supervision: Kim.

\section{Correspondence}

Seung-Ki Kim, Division of Pediatric Neurosurgery, Seoul National University Children's Hospital, Seoul National University College of Medicine, 101 Daehak-ro, Jongno-gu, Seoul 110-744, Republic of Korea. email: nsthomas@snu.ac.kr. 